\title{
Acortar los estudios de pre título en Medicina, en Chile: ¿Ahora y para todos?
}

Editor, Revista Médica de Chile.

Correspondencia a:

Dr. Humberto Reyes B.

revmedchile@smschile.cl

HUMBERTO REYES B.

\section{Shortening undergraduate medical training: now and for all medical schools in Chile?}

In Chile, undergraduate medical education starts after High School, it lasts seven years, with the final two dedicated to a rotary internship, taking to an M.D. degree that allows the graduate to enter working activities. The country needs more M.D.s in primary care, but there is also a shortage of specialists, mainly out of the main cities. In recent decades, post graduate programs leading to specialty titles have become competitively adopted by a large proportion of medical graduates. This is the case at the Pontificia Universidad Católica de Chile, stimulating its faculties and medical students to develop a collaborative review of their teaching programs, leading to a curricular reform with a new graduate profile and a new curriculum oriented to learning objectives, that will allow to obtain the M.D. degree in six instead of seven years of undergraduate education. This new program awakened expectations in other universities in Chile, that will have to face the attraction of this shortened program for future candidates to enter medical schools. However, any shortening of medical school careers should first consider the local conditions in quality of applicants, number of accepted students, the training of teachers in integrated teaching programs, the availability of adequate campuses. Furthermore, for students with different academic backgrounds and diverse personal and familial interests, the seven years programs may still be necessary to gain the expertise required to become medical doctors.

(Rev Med Chile 2016; 144: 7-10)

Key words: Curriculum; Education, medical; Professional competence.

\section{T.T.T. (Things Take Time!)}

Piet Hein (1905-1996)

E $\mathrm{n}$ el año 2014, las autoridades académicas de la Pontificia Universidad Católica de Chile $J$ (PUC) anunciaron una reforma curricular en la carrera de medicina que reduce a seis años los estudios conducentes al título de Médico Cirujano. Esta noticia fue difundida en la prensa nacional y se convirtió en un tema de interés compartido por otras universidades. ¿Por qué y para qué acortar una carrera universitaria cuya duración (siete años) se ha mantenido desde hace muchas décadas? El nuevo plan coincidió en la prensa con propuestas similares para otras profesiones, pero el caso de medicina en la PUC es ahora una realidad que conviene difundir y analizar ${ }^{1}$.

La duración de los estudios de medicina es una de las más prolongadas, universalmente. Sin embargo, no está "esculpida en piedra" y se han registrado diferencias motivadas no sólo por criterios académicos sino también, lamentablemente, por situaciones políticas y sociales, como las vividas en la Unión Soviética durante y después de la Segunda Guerra Mundial. Intentos por formar 
médicos en plazos breves, en países occidentales, han fracasado y demandaron serios esfuerzos para "nivelar" las competencias que deben dominar quienes ejercen la profesión de médicos, acorde con el nivel científico que tienen sus estudios y la experiencia clínica que deben adquirir durante su período de formación, guiados por docentes adecuados y atendiendo a pacientes reales.

En países europeos la carrera de medicina dura seis o siete años (a continuación de la enseñanza secundaria) y una de las razones para ello estriba en si el período final de práctica clínica supervisada-denominado comúnmente "internado"- se considera un requisito para otorgar el título que permite ejercer como médico, o se traspasa a un período inmediato de formación de post título en alguna especialidad médica.

En los Estados Unidos de Norteamérica, la enseñanza secundaria (High School) es seguida por un período de College, que habitualmente dura tres años, después de lo cual se inicia el período de escuela de medicina propiamente tal, con una duración generalmente de cuatro años, de modo que la edad de graduación de sus médicos no difiere en más de un año con lo que ocurre en Europa y en América Latina, incluyendo a Chile. La situación en los EE. UU. de NA se consolidó después del informe evacuado por Abraham Flexner, en 1924, quien recomendó formar médicos mediante una secuencia de asignaturas de ciencias básicas, pre clínicas y clínicas, que fue adoptada en la mayoría de los países.

Los programas de formación de médicos han estado en continua evolución, con distintas velocidades según la época y las instituciones. Han influido notoriamente las necesidades de cada país en salud pública, sus sistemas de atención médica, la disponibilidad de pacientes para la enseñanza (particularmente los "campos clínicos") y los cambios determinados por el progreso científico y tecnológico incorporados a la medicina. En nuestro país, ninguna escuela de medicina ha mantenido programas estáticos y la mayoría debió asumir cambios bruscos en la década de 1960 (período de "reforma universitaria"). A través del tiempo todas han adoptado cambios de cierta envergadura.

En años recientes, varias iniciativas regionales o nacionales han puesto en el tapete de discusión la pertinencia de acortar los estudios de pre título en distintas carreras universitarias, trasladando algunas temáticas a una formación de post título, circunscrita a una especialidad profesional. En medicina, los ejemplos más frecuentes nacieron en los Estados Unidos de Norteamérica, donde 35\% de sus escuelas de medicina acortaron de 4 a 3 años el período de formación pre clínica y clínica. En el año 2012, Emanuel y Fuchs abogaron en favor de esta medida, destinada a: 1) Reducir el costo de la carrera, que significaba para cada estudiante contraer una deuda estimada en US\$160.000, que debían cancelar durante sus estudios o después de graduarse; y 2) Aumentar el número de médicos dedicados a la atención primaria ${ }^{2}$. Un año después, Abramson et al revisaron la situación en universidades norteamericanas y canadienses, concluyendo que si bien este acortamiento era apto para estudiantes selectos, debería considerarse como sólo uno de los posibles cambios adoptables en la "era post-Flexneriana", porque el proceso de formación de un médico requiere un período extenso de aprendizaje y experiencias prácticas ${ }^{3}$. Más crítica aún fue la posición de Goldfarb y Morrison, quienes plantearon que los estudios de medicina no deberían acortarse para el estudiante "típico" o común que procura obtener el título de médico ${ }^{4}$. Estos autores recalcaron que la proporción de escuelas de medicina norteamericanas que comprimieron su plan curricular cayó de $23 \%$ en 1990 a 7\% en 2011. Hicieron hincapié en que la formación de los médicos exige no sólo el aprendizaje de conocimientos científicos sino, más aún, la adquisición de competencias (expertise) mediante una práctica que demanda tiempo, volumen de pacientes atendidos por cada alumno y un control adecuado por docentes calificados. Mientras reconocían que una proporción -estimada como pequeña-de los estudiantes tendrían las condiciones intelectuales, formación previa y dedicación necesarias para formarse adecuadamente y egresar sin que se notaran falencias en comparación con egresados de escuelas que mantenían el plan de 4 años, el problema se presentaba para la gran masa de estudiantes que resentían la intensidad de los estudios y de las prácticas clínicas, terminando su carrera exhausted. Alrededor de $25 \%$ de los alumnos encuestados y muchos docentes se manifestaron insatisfechos con el currículo abreviado. Por otra parte, la duración del programa (sea de 3 o 4 años) no parecía modificar el interés de los estudiantes por la atención primaria en salud, en lo cual influyen otros factores, tales como la percepción de poder obtener mejores rentas (sobre 
todo con el uso de tecnologías especializadas), una distinta imagen social, la mayor facilidad para ganar experiencia cuando se trabaja en un área reducida de situaciones clínicas.

El nuevo programa de la Universidad Católica de Chile no declara como objetivo primario el fundamento económico mencionado por algunas escuelas norteamericanas, sino que se basaría en condiciones detectadas en su propio ambiente de estudiantes, de académicos y de recursos para la docencia: los alumnos que ingresan a su escuela de medicina tienen una preparación previa de calidad alta y uniforme, y están motivados positivamente; el cuerpo docente ha sido preparado en metodología educacional moderna y manifiesta espíritu de pertenencia institucional, aptitud para adaptarse a los cambios y compromiso con la formación de los estudiantes; los campos clínicos son suficientes y adecuados; la institución ha puesto en práctica un sistema de autoevaluación que involucra a docentes y alumnos, permitiéndoles corregir oportunamente las deficiencias que se detecten. Y un argumento trascendental reside en que la mayor proporción de sus egresados opta de inmediato por una beca de especialización, lo cual justificaría reformular el perfil de egreso de sus graduados ${ }^{1}$.

La tendencia a preferir una formación en especialidades médicas apenas recibido el título profesional ha sido identificada en varias escuelas de medicina del país. El perfil de egreso enunciado por la mayoría de las escuelas de medicina chilenas es heterogéneo y varía desde un perfil "generalista" o de "médico integral" hasta una orientación explícita a la especialización inmediata ${ }^{5}$. En ciertos casos las declaraciones institucionales entremezclan sus intenciones con una ambigüedad difícil de entender.

En las décadas de 1950 y 1960, se esperaba que de las escuelas de medicina egresaran profesionales capacitados para actuar de inmediato como "médicos generales de zona", que trabajarían en pueblos y ciudades pequeñas, atenderían adultos y niños, y en caso de aislamiento geográfico con imposibilidad de derivación serían capaces de operar (con cirugía abierta tradicional) una apendicitis aguda, una hernia inguinal estrangulada, una cesárea, sin otro equipo humano que una enfermera o una auxiliar entrenada. Hoy, las capacidades quirúrgicas se enseñan y practican en el post título correspondiente; muchos lugares alejados de las grandes ciudades cuentan con enfermeras, matronas, nutricionistas, tecnólogos médicos, kinesiólogos, etc., que pueden constituirse en equipos para la atención en salud; hay más facilidades de comunicación, transporte y derivación oportuna; y está en sus albores el acceso a recursos de diagnóstico e interconsultas mediante telemedicina.

En el Siglo 21, los contenidos del currículo en la carrera de medicina no pueden limitarse a las materias científicas que caracterizan su profesionalidad: actualmente las competencias profesionales requieren conocimientos de psicología, habilidades comunicacionales, un enfoque social y humanista, capacidad para trabajar en grupos multi profesionales y ejercer liderazgo en ellos, fundamentos de administración de recursos humanos y materiales y, sobre todo, una ética profesional sólida. Estos elementos, que conducen a un perfil de egreso complejo, deben incorporarse paulatinamente a lo largo de la malla curricular, disponiendo de los recursos necesarios para que el alumno adquiera las competencias correspondientes, siendo fundamental el modelo de rol (role model) que le ofrecen sus docentes, particularmente en el "currículo oculto" representado por su actuar personal frente a los alumnos.

Desde el año 2008, docentes y alumnos de la escuela de medicina de la PUC han analizado su programa de estudios y diseñaron estrategias para conseguir una integración horizontal y vertical de asignaturas, enfocadas en metas de aprendizaje en "dominios terminales" la carrera de siete a seis años no sería un objetivo establecido a priori sino que habría surgido del proceso de revisión y modificación de su malla curricular. Sin duda este plan curricular será atractivo para las nuevas generaciones de egresados de la enseñanza secundaria que deseen estudiar medicina y cumplan las condiciones requeridas para postular a la escuela de medicina de la PUC.

¿Es conveniente que todas las escuelas de medicina chilenas acorten la duración de sus estudios de pre título? La respuesta está ligada a si disponen de condiciones similares en cuanto al ingreso de alumnos "top", si su número de alumnos es el adecuado para su disponibilidad de campos clínicos, la preparación docente de sus académicos, etc. Otra consideración importante es si los intereses profesionales de sus alumnos y sus expectativas laborales requieren una formación inmediata como especialistas. Las responsabilidades y compromi- 
sos familiares que contraen muchos estudiantes durante su carrera pre título, el interés por ejercer su profesión en una localidad determinada, a veces lejos de centros universitarios y sin sociedades científicas que los apoyen en un perfeccionamiento profesional continuo, pueden causar que en su desempeño futuro se note la falta de ese séptimo año que contribuye a ganar experiencia y madurez en un ambiente educacional apropiado.

La innovación curricular iniciada ya por la PUC parece sensata y estimulante. Pero creo conveniente que quienes quieran imitarla empiecen por analizar su propia realidad y después elaboren las reformas necesarias. La formación de un médico requiere procesos complejos que conducen a aprender a tomar decisiones responsables, disponer de recursos humanos y materiales para la docencia, y exige que los alumnos adquieran experiencia y madurez para manejar las situaciones que van a enfrentar con mayor frecuencia, en su ambiente laboral y en la época en que van a ejercer la profesión. Aquí aplicaría la "crítica admonición:
T.T.T." de Piet Hein, que puede traducirse por "Todo Toma Tiempo".

\section{Referencias}

1. Cisternas M, Rivera S, Sirham M, Thone M, Valdés C, Pertuzé J, et al. Reforma Curricular de la Carrera de Medicina de la Pontificia Universidad Católica de Chile. Rev Med Chile 2016; 144: 102-7.

2. Emanuel EJ, Fuchs VR. Shortening Medical Training by 30\%. JAMA 2012; 307 (11): 1143-4.

3. Abramson SB, Jacob D, Rosenfeld M, Buckvar-Keltz L, Harnik V, Francois F, et al. A 3-Year M.D. -Accelerating Careers, Diminishing Debt. N Engl J Med 2013; 369 (12): 1085-7.

4. Goldfarb S, Morrison G. The 3-Year Medical SchoolChange or Shortchange? N Engl J Med 2013; 369 (12): 1087-9.

5. Parada M, Romero MI, Moraga F. Perfiles de egreso de las carreras de Medicina en Chile. Rev Med Chile 2015; 143: 512-9. 